\title{
EFEITO DA ESTRUTURA DO CONSELHO DE ADMINISTRAÇÃO NA EVIDENCIAÇÃO DE PRÁTICAS AMBIENTAIS E SOCIAIS EM EMPRESAS BRASILEIRAS
}

\section{EFFECT OF BOARD STRUCTURE ON DISCLOSURE OF ENVIRONMENTAL AND SOCIAL PRACTICES IN BRAZILIAN COMPANIES}

\section{EFECTO DE LA ESTRUCTURA DEL CONSEJO DE ADMINISTRACIÓN EN LA EVIDENCIA DE PRÁCTICAS AMBIENTALES Y SOCIALES EN LAS EMPRESAS BRASILEÑAS}

Recebido em: 03-01-2020

Avaliado em: 10-03-2020

Reformulado em: 30-03-2020

Aceito para publicação em: 12-05-2020

Publicado em: 21-04-2021

Editor Responsável: Roberto Carlos Klann

\author{
Hyane Correia Forte ${ }^{1}$ \\ Larissa Karoline Souza Silva ${ }^{2}$ \\ Mônica Cavalcanti Sá de Abreu ${ }^{3}$
}

\section{RESUMO}

O estudo analisa a influência da estrutura do conselho de administração na evidenciação de práticas ambientais e sociais em empresas brasileiras. Baseado na teoria da agência, foram selecionadas 73 empresas listadas na Brasil, Bolsa, Balcão (B3), que divulgaram relatório de sustentabilidade GRI no formato G4, no período de 2013 a 2017. A variável dependente é a evidenciação de práticas de responsabilidade social corporativa, a qual subdivide-se em três dimensões: evidenciação total, evidenciação ambiental e evidenciação social. Quanto às variáveis independentes, o estudo utilizou a proporção de conselheiros independentes no conselho de administração, a proporção de mulheres presentes no conselho, a idade média dos membros que constituem o conselho e o número de reuniões anuais do conselho de administração. Foram estimados modelos de regressão Pooled OLS, além da análise descritiva dos dados. Observa-se um baixo percentual de mulheres no conselho de administração e a limitada diversidade geracional dos seus membros. Os resultados econométricos apontam que a proporção de conselheiros independentes no conselho de administração influencia positivamente na evidenciação de informações ambientais e sociais. Verificou-se que o número de reuniões anuais do conselho influencia negativamente, enquanto a proporção de mulheres no conselho e a idade média dos conselheiros não apresentaram significância. O estudo conclui que a composição equilibrada do conselho pode influenciar na evidenciação de práticas ambientais e sociais.

Palavras-chave: Teoria da Agência. Governança Corporativa. Evidenciação. Assimetria Informacional. Responsabilidade Social Corporativa.

\footnotetext{
1 Mestra em Administração e Controladoria no Programa de Pós-Graduação em Administração e Controladoria na Universidade Federal do Ceará (UFC); E-mail: hyane.correia@gmail.com

2 Mestra em Administração e Controladoria no Programa de Pós-Graduação em Administração e Controladoria na Universidade Federal do Ceará (UFC); E-mail: larissa.kss@ outlook.com

3 Professora Titular na Universidade Federal do Ceará - Departamento de Administração; E-mail: mabreu@ufc.br
} 


\section{ABSTRACT}

The study analyzes the board structure's influence on the disclosure of environmental and social practices in Brazilian companies. Based on Agency Theory, we selected 73 companies listed in Brazil, Bolsa, Balcão (B3) which released sustainability report GRI in G4 format, from 2013 to 2017. The dependent variable is the disclosure of corporate social responsibility practices, which is subdivided into three dimensions: total, environmental and social disclosure. Regarding the independent variables, the study used the proportion of independent board members, the proportion of women on the board, the board members' average age, and the number of annual board meetings. Pooled OLS regression models were estimated, as well as descriptive data analysis. There is a low percentage of women on the board and the limited generational diversity of its members. The econometric results indicate that the proportion of independent directors on the board of directors positively influences the environmental and social information disclosure. The number of annual board meetings was found to have a negative influence, while the proportion of women on the board and the board members' average age were not significant. The study concludes that the board's balanced composition can influence the disclosure of environmental and social practices.

Keywords: Agency Theory. Corporate Governance. Disclosure. Informational Asymmetry. Corporate Social Responsibility.

\section{RESUMEN}

El estudio analiza la influencia de la estructura del directorio en la divulgación de prácticas ambientales y sociales en empresas brasileñas. Con base en la Teoría de la agencia, se seleccionaron 73 empresas que cotizan en Brasil, Bolsa, Balcão (B3), que publicaron el informe de sostenibilidad GRI en formato G4, durante el período de 2013 a 2017. La variable dependiente es la divulgación de prácticas de responsabilidad social corporativa, que se subdivide en tres dimensiones: divulgación total, divulgación ambiental y divulgación social. Con respecto a las variables independientes, el estudio utilizó la proporción de miembros independientes de la junta, la proporción de mujeres en la junta, la edad promedio de los miembros de la junta y el número de reuniones anuales de la junta. Se estimaron modelos de regresión de OLS agrupados, así como análisis de datos descriptivos. Hay un bajo porcentaje de mujeres en la junta y la limitada diversidad generacional de sus miembros. Los resultados econométricos indican que la proporción de directores independientes en la junta directiva influye positivamente en la divulgación de información ambiental y social. Se encontró que el número de reuniones anuales de la junta tenía una influencia negativa, mientras que la proporción de mujeres en la junta y la edad promedio de los miembros de la junta no eran significativas. El estudio concluye que la composición equilibrada de la junta puede influir en la divulgación de prácticas ambientales y sociales.

Palabras clave: Teoría de la Agencia. Gobierno Corporativo. Divulgación. Asimetría Informacional. Responsabilidad Social Corporativa.

\section{INTRODUÇÃO}

A evidenciação de práticas de responsabilidade social corporativa (RSC) pode ser influenciada pela composição do conselho de administração (Giannarakis, 2014). O conselho de administração monitora a relação entre o "agente" e o "principal", e influencia o direcionamento de políticas e estratégias corporativas (Muttakin, Khan, \& Mihret, 2018). Exerce relevante papel na avaliação das operações (Naciti, 2019) e na proteção dos interesses apropriados das partes interessadas e, consequentemente, reduz os problemas de agência (Hu \& Loh, 2018; Said, Zainuddin, \& Haron, 2009). A diversidade no conselho de administração vai além da heterogeneidade de seus 
membros. Envolve diferentes percepções, valores e particularidades, gerados a partir da convivência dos seus integrantes, e contribui para as tomadas de decisões em benefício da empresa.

Estudos sugerem que um conselho de administração com maior presença de conselheiros independentes, com uma maior diversidade de gênero, maior faixa etária e mais ativo pode influenciar a evidenciação de práticas ambientais e sociais (Rao \& Tilt, 2016). O conselho de administração é, portanto, um importante mecanismo de controle interno de governança corporativa e necessita de uma estrutura equilibrada, para que as questões sociais e ambientais sejam gerenciadas, e os riscos controlados (Helfaya \& Moussa, 2017; Hu \& Loh, 2018; Jizi, Salama, Dixon, \& Stratling, 2014; Modiba \& Ngwakwe, 2017). À medida que o conselho de administração apresenta-se mais diversificado, orienta uma conduta mais efetiva de seus membros, proporciona melhor compreensão sobre as demandas do mercado e das suas partes interessadas. Consequentemente, possibilita uma adequada tomada de decisão (Torchia, Calabrò, Huse, \& Brogi, 2010).

O IBGC (2015) orienta que um conselho de administração mais diversificado permite maior conjunto de informações e levantamento de questões sobre a empresa, bem como maior congruência dos valores organizacionais com as questões sociais e ambientais. Pucheta-Martínez, Bel-Oms e Olcina-Sempere (2018a) alertam que estas vantagens não são possíveis com a formação de um conselho com características tradicionais. De acordo com o IBGC (2016), 22,7\% das empresas listadas na bolsa de valores brasileira possuem conselheiros independentes, apenas 7,9\% garantem a presença de mulheres no conselho de administração, e 70,1\% dos conselheiros têm uma idade média entre 50 a 70 anos. Existe, portanto, um desafio em diversificar o conselho de administração e, assim, de evoluir no ambiente de negócios.

Identifica-se, portanto, a necessidade de investigar a composição do conselho de administração de empresas brasileiras, no que tange a sua influência na evidenciação de práticas de RSC. O artigo toma por base a seguinte pergunta de pesquisa: Em que extensão a estrutura do conselho de administração está relacionada à evidenciação de práticas ambientais e sociais? A pesquisa traz contribuições para a área da governança corporativa. Primeiro, a diversidade no conselho de administração pode beneficiar as corporações na tomada de decisões (García-Sánchez, Suárez-Fernández, \& Martínez-Ferrero, 2019). Em segundo, a estrutura do conselho de administração pode afetar (ou não) a elaboração dos relatórios de RSC e, consequentemente, a implantação de práticas ambientais e sociais. Por fim, destaca-se que pesquisas sobre composição do conselho de administração em países emergentes, como o Brasil, ainda são incipientes (Dani, Picolo, \& Klann, 2019).

Com base no exposto, a próxima seção apresenta as hipóteses sobre a influência do conselho de administração e evidenciação de práticas ambientais e sociais. Em seguida, apresenta-se a metodologia adotada para coletar dados em 73 empresas listadas na B3, que divulgaram relatórios de sustentabilidade de 2013 a 2017. Tomando por base os dados coletados, foram estimados modelos de regressão do tipo Pooled OLS em um painel de dados. Finalmente, são apresentados e discutidos os resultados da pesquisa, os quais reforçam a importância da diversidade do conselho de administração para evidenciação de RSC.

\section{DIVERSIDADE DO CONSELHO DE ADMINISTRAÇÃO E EFEITOS NA EVIDENCIAÇÃO DE PRÁTICAS AMBIENTAIS E SOCIAIS}

Empresas devem equilibrar a pluralidade de interesses das partes interessadas por meio da definição de políticas e práticas de RSC (Jizi et al., 2014; Van Marrewijk, 2003, Jamali, Safieddine, \& Rabbath, 2008; Rao \& Tilt, 2016) e evidenciá-las nos relatórios de sustentabilidade (Guidry \& Patten, 2010; Hu \& Loh, 2018; Reverte, 2016). A divulgação de informações não financeiras é, portanto, um mecanismo de controle que minimiza a assimetria informacional e atenua os problemas de agência (Harjoto \& Jo, 2011; Pucheta-Martínez, Bel-Oms, \& Olcina-Sempere, 2018b). 
Empresas que se preocupam com questões sociais e ambientais, além das econômicas e financeiras, podem estabelecer relações de confiança com suas partes interessadas, além de criar valor (Harrison, Bosse, \& Phillips, 2010). Uma adequada governança corporativa permite que os gestores assumam compromissos e solucionem os conflitos entre "atender demandas das partes interessadas" e "maximizar a riqueza dos acionistas" (De La Cuesta \& Valor, 2013). O aumento do valor de mercado pode ser atingido por meio de práticas de RSC e a propagação de uma imagem positiva da empresa (Fernández-Gago, Cabeza-García, \& Nieto, 2016). A transparência é, portanto, fundamental para dar legitimidade às práticas de RSC (Font, Walmsley, Cogotti, McCombes, \& Häusler, 2012).

A estrutura do conselho de administração está associada aos seguintes atributos: presença de conselheiros independentes; mulheres no quadro do conselho de administração; conselheiros jovens ou experientes; e conselheiros mais comprometidos com a empresa. A teoria da agência considera que a diferenciação na composição do conselho de administração, com a presença de conselheiros internos e independentes, resulta em um melhor monitoramento das atividades corporativas (Basuony, Mohamed, \& Samaha, 2018). Considera-se "conselheiros independentes" aqueles externos que não possuem ligação com a empresa, seja de ordem familiar, por meio de negócios, e/ou relação com sócios que possuem significativa participação acionária. Conselheiros independentes devem evitar julgamentos ou decisões comprometidas, e atuar no sentido de tomar decisões em benefício da empresa (IBGC, 2015). A maior presença de conselheiros independentes no conselho de administração favorece a governança corporativa e a realização de atividades que consideram os pleitos das partes interessadas e dos acionistas (Jizi, 2017).

Muttakin, Khan e Mihret (2018) afirmam que uma maior proporção de conselheiros independentes e o tamanho do conselho de administração causam impactos positivos na evidenciação de práticas de RSC. Nesse sentido, o aumento da legitimidade dos relatórios de RSC pode influenciar em um melhor desempenho financeiro da empresa (Fernández-Gago et al., 2016). A presença de conselheiros independentes no conselho favorece, portanto, o adequado direcionamento dos recursos financeiros, com o intuito de potencializar a relevância de iniciativas sociais e ambientais, e a posterior evidenciação (Jizi, 2017). Dessa forma, formula-se a primeira hipótese da pesquisa:

Hipótese 1: Uma maior proporção de conselheiros independentes no conselho de administração influencia positivamente a evidenciação de práticas ambientais e sociais.

A diversidade de gênero no conselho de administração pode influenciar a adoção de práticas discricionárias de RSC (Bear \& Rahman; Post, 2010; Rao \& Tilt, 2016). Trata-se de um aspecto demográfico, o qual se refere a proporção de homens e mulheres na "sala de reuniões" (Campbell \& Mínguez-Vera, 2008). A diversidade demográfica reflete o ambiente socioeconômico e pode favorecer a compreensão da complexidade do ambiente de negócios (Hillman \& Dalziel, 2003). A presença de mulheres no conselho de administração pode trazer um "outro olhar" na mesa de negociações. Mulheres tendem a ter seus valores alinhados com atividades filantrópicas e são mais voltados ao bem-estar da sociedade do que aos seus próprios interesses (Jizi, 2017).

Amran, Lee e Devi (2014) afirmam que na ausência de diversidade de gênero, a empresa não dispõe de capacidades distintas e conhecimentos necessários para adotar práticas de RSC, com impactos na transparência e na evidenciação. Uma maior participação de mulheres no conselho de administração pode, portanto, sensibilizar as iniciativas de RSC e, por consequência, impactar no desempenho social corporativo (Bear, Rahman, \& Post, 2010; Hafsi \& Turgut, 2013). A presença de mulheres na composição do conselho de administração pode encorajar a elaboração de relatórios de sustentabilidade, com foco no bem-estar das partes interessadas e, por consequência, melhorar a imagem corporativa (Jizi, 2017). A maior sensibilidade das mulheres com relação às práticas de RSC pode contribuir para o melhor relacionamento e comunicação da empresa com suas partes interessadas. Diante do exposto, sugere-se a segunda hipótese da pesquisa: 
Hipótese 2: Uma maior proporção de mulheres no conselho de administração influencia positivamente a evidenciação de práticas ambientais e sociais.

Além da diversidade de gênero, outro aspecto importante na evidenciação de práticas ambientais e sociais envolve a diversidade etária dos membros que compõe o conselho de administração (Rao \& Tilt, 2016). A idade dos integrantes da alta administração reflete a maturidade dos executivos frente aos negócios e pode influenciar nas estratégias de RSC (Hafsi \& Turgut, 2013). A idade relaciona-se à conduta dos conselheiros e sua receptividade a novas ideias (Zajac \& Westphal, 1996). Também refletem diferentes valores, motivações e interesses no ambiente de trabalho (Ferrero-Ferrero, Fernández-Izquierdo, \& Muñoz-Torres, 2013). Post, Rahman e Rubow (2011) enfatizam que a idade dos membros do conselho de administração exerce influência sobre as decisões filantrópicas da organização.

Experiência e conhecimento dos gestores de maior idade e a dinâmica dos mais novos são relevantes no processo de tomada de decisão (Rao \& Tilt, 2016). Membros do conselho de administração com idade mais elevada estão mais inclinados ao bem-estar da sociedade, enquanto os mais jovens apresentam-se como mais sensíveis às questões ambientais e éticas (Hafsi \& Turgut, 2013). Post et al. (2011) argumentam que conselheiros mais experientes podem, também, ser propensos a implementar processos de governança ambiental. Membros do conselho de administração mais experientes, ou seja, com uma faixa etária mais elevada, tendem a empreender políticas e estratégias de RSC, favorecendo a maior preocupação com os aspectos sociais e ambientais. Assim, são mais propensos a apresentar um maior relacionamento e comunicação com a comunidade, investidores e demais partes interessadas. Dessa forma, frente aos argumentos apresentados, elaborou-se a terceira hipótese:

Hipótese 3: Uma maior idade média dos membros do conselho de administração influencia positivamente a evidenciação de práticas ambientais e sociais.

Dienes e Velte (2016) relatam que a pesquisa empírica é inconclusiva no que concerne ao impacto da atividade do conselho de administração, avaliado por meio do número de reuniões ao longo do ano. No entanto, argumenta-se que o aumento no número de reuniões pode ser entendido como ocasiões de eminentes riscos aos interesses dos acionistas ou situações de decisões conflitantes (Ahmad, Rashid, \& Gow, 2017; Liang, Xu, \& Jiraporn, 2013). Um número progressivo de reuniões não resulta, necessariamente, em um padrão elevado de governança corporativa, além de representar um alto custo. Contudo, em cenários de desastres ambientais é inevitável uma maior frequência de reuniões do conselho de administração, para auxiliar no posicionamento apropriado para mitigar os danos à sociedade e os impactos negativos à empresa (Dienes \& Velte, 2016).

As reuniões do conselho de administração não devem focar nas atividades do dia-a-dia da empresa. Ao contrário, devem envolver conteúdos fundamentais e estratégias que tenham o potencial de aumentar o desempenho corporativo (Ahmad et al., 2017). Adicionalmente, um elevado número de reuniões do conselho de administração deve ser evidenciado no relatório de sustentabilidade, e pode ter impacto na confiança dos acionistas (Ahmad et al., 2017; Vafeas, 1999). Jensen (1993) alega que o aumento das atividades do conselho de administração pode sinalizar más notícias para as partes interessadas. Em geral, o conselho de administração fica relativamente inativo em empresas com desempenho financeiro e social estável. Nesse contexto, propõe-se a quarta hipótese da pesquisa:

Hipótese 4: Uma maior atividade do conselho de administração influencia negativamente a evidenciação de práticas ambientais e sociais. 


\section{METODOLOGIA}

A pesquisa apresenta abordagem quantitativa, exploratória e descritiva. Foram selecionadas todas as 73 empresas listadas na B3 S.A (Brasil, Bolsa, Balcão) que divulgaram relatórios de RSC, com base nas diretrizes da Global Reporting Initiative (GRI) no formato G4, no período compreendido entre os anos de 2013 até 2017. Destaca-se que as diretrizes do GRI-G4 foram desenvolvidas para serem aplicáveis às organizações de todos os tamanhos e setores (GRI, 2015). Ao total, foram coletadas 190 observações, em que 16,4\% das empresas fazem parte do setor financeiro, $50,4 \%$ do setor industrial e $30,2 \%$ do setor de serviços.

A mensuração da evidenciação de informações de caráter ambiental e social seguiu a proposta metodológica desenvolvida por Fischer e Sawczyn (2013), mas utilizou os indicadores de desempenho do GRI (2013). Soares et al. (2018a) e Soares et al. (2018b) também utilizaram estes indicadores para construírem as variáveis que tratam da evidenciação total (EVT), evidenciação ambiental (EVA) e evidenciação social (EVS). Conforme estabelecido por Fischer e Sawczyn (2013), cada indicador de desempenho foi pontuado de 0 a 6 , conforme os seguintes critérios: 0 quando não é divulgada informação; 1 quando a informação de desempenho é apresentada em termos absolutos ou relativos. Soma-se mais 1 ponto quando, além da informação em termos absolutos ou relativos, ela também é dada: em relação a empresas semelhantes/rivais ou setor; em comparação com períodos passados; em relação às metas estipuladas; de forma normalizada ou de forma desagregada. As características avaliadas para cada indicador são apresentadas na Tabela 1.

Tabela 1 - Características avaliadas nos indicadores ambientais e sociais

\begin{tabular}{lc}
\multicolumn{1}{c}{ Características } & Valores \\
\hline Indicador ausente & 0 \\
Informação absoluta ou relativa de desempenho apresentada & 1 \\
A informação é apresentada e comparada com outras empresas do setor. & +1 \\
A informação é apresentada e comparada com períodos passados & +1 \\
A informação é apresentada e comparada com metas traçadas & +1 \\
A informação é apresentada também de forma normalizada & +1 \\
A informação é apresentada de forma desagregada & +1 \\
Pontuação máxima por indicador & 6 \\
\hline
\end{tabular}

Fonte: Fischer e Sawczyn (2013).

A evidenciação ambiental é constituída de 9 indicadores e a pontuação máxima obtida por empresa é de 54 pontos, conforme apresentado na Tabela 2.

Tabela 2 - Indicadores do GRI-G4 utilizados para avaliar a evidenciação ambiental

\begin{tabular}{|c|l|}
\hline $\begin{array}{c}\text { Indicador de } \\
\text { Desempenho }\end{array}$ & \multicolumn{1}{c|}{ Evidenciação Ambiental - Pontuação Máxima: 54 pontos } \\
\hline EN2 & Pescrição \\
\hline EN3/EN4 & Consumo de energia dentro/fora da organização. \\
\hline EN8/EN22 & Total de retirada de água e descarte total de água, por fonte/destinação. \\
\hline EN11/EN12 & $\begin{array}{l}\text { Descrição de impactos significativos de atividades, produtos e serviços sobre a biodiversidade em } \\
\text { áreas protegidas e áreas de alto índice de biodiversidade situadas fora de áreas protegidas. }\end{array}$ \\
\hline EN15/EN16/ & $\begin{array}{l}\text { Emissões diretas e indiretas de gases de efeito estufa (GEE) (Emissão de substâncias que destroem } \\
\text { a camada de ozônio, NOx, SOx e outras emissões atmosféricas significativas), por peso. }\end{array}$ \\
\hline EN17/EN20/EN21 & Peso total de resíduos, discriminado por tipo e método de disposição. \\
\hline EN23 & Extensão da mitigação de impactos ambientais de produtos e serviços. \\
\hline EN28 & $\begin{array}{l}\text { Percentual de produtos e suas embalagens recuperados em relação ao total de produtos vendidos, } \\
\text { discriminados por categoria de produtos. }\end{array}$ \\
\hline EC2 & $\begin{array}{l}\text { Implicações financeiras e outros riscos e oportunidades para as atividades da organização em } \\
\text { decorrência de mudanças climáticas. }\end{array}$ \\
\hline
\end{tabular}

Fonte: Soares et al. (2018a) e Soares et al. (2018b). 
Para as categorias que apresentam mais de um indicador, cada indicador foi avaliado separadamente e considerado a maior pontuação entre eles.

A evidenciação social é formada por 14 indicadores, em que a pontuação máxima a ser alcançada para cada empresa é de 84 pontos, como pode ser observado na Tabela 3 . De forma semelhante aos indicadores ambientais, quando as categorias apresentam mais de um indicador, cada indicador foi avaliado separadamente e considerado a maior pontuação.

Tabela 3 - Indicadores do GRI-G4 usados para avaliar a evidenciação social

\begin{tabular}{|c|c|}
\hline \multicolumn{2}{|r|}{ Evidenciação Social - Pontuação Máxima: 84 pontos } \\
\hline $\begin{array}{l}\text { Indicador de } \\
\text { Desempenho }\end{array}$ & Descrição \\
\hline LA1 & $\begin{array}{l}\text { Discriminação do número total de empregados por contrato de trabalho e gênero, de empregados } \\
\text { permanentes por tipo de emprego e gênero e por região e gênero e número total e taxas de novas } \\
\text { contratações de empregados e rotatividade de empregados por faixa etária, gênero. }\end{array}$ \\
\hline LA6 & $\begin{array}{l}\text { Tipos e taxas de lesões, doenças ocupacionais, dias perdidos, absenteísmo e número de óbitos } \\
\text { relacionados ao trabalho, discriminados por região e gênero. }\end{array}$ \\
\hline LA9 & $\begin{array}{l}\text { Número médio de horas de treinamento por ano por empregado, discriminado por gênero e categoria } \\
\text { funcional. }\end{array}$ \\
\hline LA12 & $\begin{array}{l}\text { Composição dos grupos responsáveis pela governança e discriminação de empregados por categoria } \\
\text { funcional, de acordo com gênero, faixa etária, minorias e outros indicadores de diversidade. }\end{array}$ \\
\hline LA13 & $\begin{array}{l}\text { Razão matemática do salário e remuneração entre mulheres e homens, discriminada por categoria } \\
\text { funcional e unidades operacionais relevantes. }\end{array}$ \\
\hline EC3 & Cobertura das obrigações previstas no plano de pensão de benefício da organização. \\
\hline HR3 & o total de casos de discriminação e medidas corretivas tomadas. \\
\hline HR5 & $\begin{array}{l}\text { Operações e fornecedores identificados como de risco para a ocorrência de casos de trabalho infantil } \\
\text { e medidas tomadas para contribuir para a efetiva erradicação do trabalho infantil. }\end{array}$ \\
\hline HR6 & $\begin{array}{l}\text { Operações e fornecedores identificados como de risco significativo para a ocorrência de trabalho } \\
\text { forçado ou análogo ao escravo e medidas tomadas para contribuir para a eliminação de todas as formas } \\
\text { de trabalho forçado ou análogo ao escravo. }\end{array}$ \\
\hline $\mathrm{SO} 3 / \mathrm{SO} 4$ & $\begin{array}{l}\text { Número total e percentual de operações submetidas a avaliações de riscos relacionados à corrupção e } \\
\text { os riscos significativos identificados e comunicação e treinamento em políticas e procedimentos de } \\
\text { combate à corrupção. }\end{array}$ \\
\hline EC7 & Desenvolvimento e impacto de investimentos em infraestrutura e serviços oferecidos. \\
\hline EC9 & Proporção de gastos com fornecedores locais em unidades operacionais importantes. \\
\hline PR2 & $\begin{array}{l}\text { Número total de casos de não conformidade com regulamentos e códigos voluntários relacionados aos } \\
\text { impactos causados por produtos e serviços na saúde e segurança durante seu ciclo de vida, } \\
\text { discriminados por tipo de resultado. }\end{array}$ \\
\hline G4 - PR3 & $\begin{array}{l}\text { Tipo de informações sobre produtos e serviços exigidos pelos procedimentos da organização } \\
\text { referentes a informações e rotulagem de produtos e serviços e percentual de categorias significativas } \\
\text { sujeitas a essas exigências. }\end{array}$ \\
\hline & 138 \\
\hline
\end{tabular}

Fonte: Soares et al. (2018a) e Soares et al. (2018b).

A evidenciação total corresponde a soma das evidenciações ambiental e social e pode alcançar 138 pontos. As variáveis dependentes da pesquisa foram mensuradas por meio da divisão entre a pontuação obtida pela empresa e a pontuação máxima da respectiva dimensão.

A Tabela 4 apresenta o constructo da Responsabilidade Social Corporativa, que envolve as variáveis dependentes - Evidenciação Total (EVT), Evidenciação Ambiental (EVA) e Evidenciação Social (EVS) -, a operacionalização do cálculo destas variáveis e a fonte da pesquisa.

Para construção das variáveis independentes, foram selecionados indicadores capazes de representar a estrutura e a diversidade do conselho de administração. De modo complementar, foram selecionadas variáveis de controle, tamanho e alavancagem, ligadas às características financeiras das empresas, além do tamanho do conselho de administração, setor de atividade e ano da observação. Essa inclusão baseia-se na ideia proposta por Roberts (1992), de que a propensão para que uma empresa adote práticas de RSC dependente da sua disponibilidade de recursos financeiros. A Tabela 
5 apresenta as variáveis independentes e de controle, a operacionalização do cálculo e as fontes de coleta de dados.

Tabela 4 - Descrição e operacionalização das variáveis dependentes

\begin{tabular}{|c|c|c|c|}
\hline Constructo & Variáveis Dependentes & Operacionalização & Fonte da Pesquisa \\
\hline \multirow{3}{*}{$\begin{array}{c}\text { Responsabilidade } \\
\text { Social } \\
\text { Corporativa } \\
\text { (RSC) }\end{array}$} & $\begin{array}{l}\text { Evidenciação de informações de } \\
\text { caráter ambiental e social (EVT) }\end{array}$ & $\frac{\text { Pontuação total obtida pela empresa }}{\text { Pontuação máxima - Dimensão Total }}$ & $\begin{array}{l}\text { Global Reporting } \\
\text { Initiative }\end{array}$ \\
\hline & $\begin{array}{l}\text { Evidenciação de informações de } \\
\text { caráter ambiental (EVA) }\end{array}$ & $\begin{array}{l}\text { Pontuação ambiental obtida pela empresa } \\
\text { Pontuação máxima - Dimensão Ambiental }\end{array}$ & $\begin{array}{l}\text { Global Reporting } \\
\text { Initiative }\end{array}$ \\
\hline & $\begin{array}{l}\text { Evidenciação de informações de } \\
\text { caráter social (EVS) }\end{array}$ & $\frac{\text { Pontuação social obtida pela empresa }}{\text { Pontuação máxima - Dimensão Social }}$ & $\begin{array}{l}\text { Global Reporting } \\
\text { Initiative }\end{array}$ \\
\hline
\end{tabular}

Fonte: Elaborado a partir de Soares et al. (2018a) e Soares et al. (2018b).

Tabela 5 - Variáveis independentes e de controle, operacionalização e fontes

\begin{tabular}{|c|c|c|c|}
\hline Constructo & Variáveis & Operacionalização & $\begin{array}{l}\text { Fonte de } \\
\text { Pesquisa }\end{array}$ \\
\hline \multirow{4}{*}{$\begin{array}{l}\text { Composição do } \\
\text { Conselho de } \\
\text { Administração } \\
\text { (COMP_CADM) }\end{array}$} & $\begin{array}{l}\text { Proporção de conselheiros } \\
\text { independentes no Conselho de } \\
\text { Administração (INDEP) }\end{array}$ & $\frac{\text { Número de conselheiros independentes }}{\text { Tamanho do conselho de administração }}$ & $\begin{array}{l}\text { Formulário de } \\
\text { Referência }\end{array}$ \\
\hline & $\begin{array}{l}\text { Proporção de mulheres no Conselho } \\
\text { de Administração (MLR_CADM) }\end{array}$ & $\begin{array}{c}\text { Número de mulheres no } \\
\text { conselho de administração } \\
\text { Tamanho do conselho de administração }\end{array}$ & $\begin{array}{l}\text { Formulário de } \\
\text { Referência }\end{array}$ \\
\hline & $\begin{array}{l}\text { Idade média do Conselho de } \\
\text { Administração (IM_CADM) }\end{array}$ & $\begin{array}{c}\sum_{\text {conselheiro de administração }} \\
\text { Tamanho do conselho de administração }\end{array}$ & $\begin{array}{l}\text { Formulário de } \\
\text { Referência }\end{array}$ \\
\hline & $\begin{array}{l}\text { Atividade do Conselho de } \\
\text { Administração (ATIV_CADM) }\end{array}$ & $\begin{array}{c}\text { Número de reuniões anuais do Conselho } \\
\text { de Administração }\end{array}$ & B3 S.A. \\
\hline \multirow{5}{*}{$\begin{array}{l}\text { Variáveis de } \\
\text { Controle } \\
(\text { CONT })\end{array}$} & $\begin{array}{l}\text { Tamanho do Conselho de } \\
\text { Administração (TAM_CADM) }\end{array}$ & $\begin{array}{l}\text { Número de membros do Conselho de } \\
\text { Administração }\end{array}$ & $\begin{array}{c}\text { Formulário de } \\
\text { Referência }\end{array}$ \\
\hline & Tamanho da empresa (TAM) & $\ln$ (Ativo total) & Economática ${ }^{\circledR}$ \\
\hline & Alavancagem (ALAV) & $\frac{\text { Dívida total }}{\text { Ativo total }}$ & Economática ${ }^{\circledR}$ \\
\hline & Setor de atividade (SETOR) & $\begin{array}{c}\text { Setor de atividade da empresa (Variável } \\
\text { dummy) }\end{array}$ & Economática ${ }^{\circledR}$ \\
\hline & Período de tempo (ANO) & Ano da observação (Variável dummy) & - \\
\hline
\end{tabular}

Fonte: Elaborado a partir dos dados da pesquisa.

A partir das variáveis elencadas, para se investigar a possível influência da composição do conselho de administração na evidenciação de práticas de RSC, foi definido o seguinte modelo econométrico:

$$
\begin{aligned}
\mathrm{RSC}_{\mathrm{it}}=\beta_{0}+\beta_{1} \mathrm{COMP}+ & \mathrm{CADM}_{\mathrm{it}}+\beta_{2} \mathrm{TAM}_{-} \mathrm{CADM}_{\mathrm{it}}+\beta_{3} \mathrm{TAM}_{\mathrm{it}}+\beta_{4} \mathrm{ALAV}_{\mathrm{it}}+ \\
& +\beta_{5} \mathrm{SETOR}_{\mathrm{it}}+\beta_{6} \mathrm{ANO}_{\mathrm{it}}+\varepsilon_{\mathrm{it}}
\end{aligned}
$$

O modelo econométrico, além de verificar a evidenciação total (EVT), apresenta um desdobramento para a evidenciação ambiental (EVA) e para a evidenciação social (EVS). Os dados coletados, a partir das 190 observações das 73 empresas listadas na B3, foram analisadas por meio de modelo de regressão múltipla do tipo Pooled OLS. Também foram avaliadas as medidas de tendência central e de dispersão. Todas as análises descritivas e econométricas foram executadas pelo software Stata versão 13. 


\section{APRESENTAÇÃO E ANÁLISE DOS RESULTADOS}

A Tabela 6 apresenta a quantidade de observações por ano e setor. Verifica-se que os anos de 2013 e 2017 apresentaram um desequilíbrio no número de observações, quando comparados com o período 2014 a 2016. Esta discrepância pode estar relacionada com ano de 2013, quando foram divulgadas as diretrizes do relatório GRI no formato G4, o qual teve pequena adesão das empresas brasileiras. Em 2017, a GRI publicou o formato Standards e algumas empresas listadas na B3 passaram a elaborar seus relatórios neste formato. Consequentemente, houve uma redução na evidenciação de relatórios GRI no formato G4. Destaca-se, ainda, que as empresas classificadas no setor de utilidade pública foram as que mais divulgaram relatórios de RSC.

Tabela 6 - Número de observações por ano e setor

\begin{tabular}{ccccccc}
\hline Setor & $\mathbf{2 0 1 3}$ & $\mathbf{2 0 1 4}$ & $\mathbf{2 0 1 5}$ & $\mathbf{2 0 1 6}$ & $\mathbf{2 0 1 7}$ & Total \\
\hline Bens industriais & 1 & 7 & 8 & 5 & 1 & 22 \\
Consumo cíclico & 5 & 7 & 10 & 8 & 1 & 31 \\
Consumo não cíclico & 2 & 4 & 6 & 4 & 0 & 16 \\
Financeiro e outros & 8 & 5 & 7 & 8 & 1 & 29 \\
Materiais básicos & 4 & 6 & 2 & 3 & 0 & 15 \\
Petróleo, gás e biocombustíveis & 2 & 3 & 4 & 4 & 1 & 14 \\
Saúde & 0 & 0 & 1 & 2 & 0 & 3 \\
Tecnologia da informação & 2 & 1 & 0 & 0 & 0 & 3 \\
Telecomunicações & 0 & 1 & 2 & 2 & 1 & 6 \\
Utilidade Pública & 6 & 12 & 12 & 15 & 6 & 51 \\
\hline Total & $\mathbf{3 0}$ & $\mathbf{4 6}$ & $\mathbf{5 2}$ & $\mathbf{5 1}$ & $\mathbf{1 1}$ & $\mathbf{1 9 0}$ \\
\hline
\end{tabular}

Fonte: Dados da pesquisa.

Tabela 7 - Análise descritiva das variáveis independentes

\begin{tabular}{cccccccc}
\hline Conselho de Administração & Ano & Média & Desvio Padrão & Mínimo & Mediana & Máximo & Moda \\
\hline & 2013 & 0,26 & 0,19 & 0,00 & 0,24 & 0,67 & 0,00 \\
Proporção de conselheiros & 2014 & 0,26 & 0,20 & 0,00 & 0,22 & 0,86 & 0,00 \\
independentes & 2015 & 0,30 & 0,25 & 0,00 & 0,25 & 0,88 & 0,00 \\
(INDEP) & 2016 & 0,29 & 0,26 & 0,00 & 0,25 & 1,00 & 0,00 \\
& 2017 & 0,41 & 0,29 & 0,00 & 0,43 & 0,86 & 0,00 \\
\hline & 2013 & 0,06 & 0,09 & 0,00 & 0,00 & 0,31 & 0,00 \\
Proporção de mulheres no & 2014 & 0,07 & 0,09 & 0,00 & 0,00 & 0,33 & 0,00 \\
conselho de administração & 2015 & 0,06 & 0,08 & 0,00 & 0,00 & 0,29 & 0,00 \\
(MLR_CADM) & 2016 & 0,10 & 0,10 & 0,00 & 0,08 & 0,43 & 0,00 \\
& 2017 & 0,07 & 0,09 & 0,00 & 0,06 & 0,29 & 0,00 \\
\hline & 2013 & 54,70 & 5,94 & 42,00 & 54,40 & 66,60 & 42,00 \\
Idade média dos membros do & 2014 & 55,60 & 6,36 & 43,20 & 54,90 & 67,10 & 43,20 \\
conselho de administração & 2015 & 56,00 & 6,16 & 43,20 & 55,60 & 67,60 & 45,70 \\
(IM_CADM) & 2016 & 56,70 & 5,90 & 39,40 & 57,10 & 68,30 & 50,00 \\
& 2017 & 57,80 & 7,36 & 43,30 & 58,60 & 70,40 & 43,30 \\
\hline & 2013 & 18,20 & 16,80 & 5,00 & 15,50 & 101,00 & 18,00 \\
Número de reuniões do conselho & 2014 & 16,30 & 8,78 & 3,00 & 15,00 & 49,00 & 10,00 \\
de administração & 2015 & 17,00 & 8,69 & 7,00 & 16,50 & 51,00 & 11,00 \\
(ATIV_CADM) & 2016 & 15,80 & 8,48 & 1,00 & 14,00 & 40,00 & 14,00 \\
& 2017 & 16,00 & 7,69 & 7,00 & 14,00 & 31,00 & 9,00 \\
\hline
\end{tabular}

Fonte: Dados da pesquisa.

A Tabela 7 traz a composição do conselho de administração segregada por ano. Observa-se que a média da proporção de conselheiros independentes apresenta um crescimento, principalmente no ano de 2017, contudo, este aumento pode estar relacionado com o baixo número de observações neste ano. As medianas são superiores a $20 \%$, ou seja, $50 \%$ ou mais das observações tem mais de $20 \%$ do conselho de administração formado por conselheiros independentes. Destaca-se que a 
proporção de conselheiros independentes apresenta desvios padrão inferiores à média, o que indica a presença de dados homogêneos.

Frente à diversidade de gênero no conselho de administração, extrai-se que, no ano de 2016, o conselho de administração tinha a média de proporção de mulheres igual a 10\%. Pode-se verificar, ainda, que no período 2016-2017, a mediana do número de mulheres foi superior a zero, ou seja, 50\% ou mais das observações tem alguma mulher compondo o conselho de administração. Porém, observa-se que a moda da proporção de mulheres é igual a zero, ou seja, a maioria das observações não têm mulheres compondo o conselho de administração.

Relativo à idade média dos membros do conselho de administração, percebe-se que a média etária é superior a 50 anos e apresentou um pequeno aumento durante o período de análise. Verificase que a mediana do período apresenta um equilíbrio. Com relação a atividade do conselho de administração, medida pelo número de reuniões, observa-se que apesar da média anual apresentar uma queda, apresentou uma variação pequena do número de reuniões. De acordo com o cálculo do desvio padrão, pode-se inferir a homogeneidade dos dados.

A Tabela 8 apresenta a estatística descritiva relativa aos índices de evidenciação das práticas ambientais, sociais e total, segregados por ano. Pode-se observar a baixa média de EVA e EVS. Contudo, as informações relativas aos indicadores de dimensão ambiental apresentaram uma média maior de evidenciação, se comparadas com a dimensão social.

Tabela 8 - Análise descritiva das variáveis dependentes

\begin{tabular}{ccccccc}
\hline Dimensão de RSC & Ano & Média & Desvio Padrão & Mínimo & Mediana & Máximo \\
\hline & 2013 & 0,1310 & 0,1130 & 0,0000 & 0,1020 & 0,3330 \\
Evidenciação Ambiental & 2014 & 0,1740 & 0,1080 & 0,0000 & 0,1670 & 0,4070 \\
(EVA) & 2015 & 0,1570 & 0,1020 & 0,0000 & 0,1480 & 0,3890 \\
& 2016 & 0,1530 & 0,0995 & 0,0000 & 0,1670 & 0,4070 \\
& 2017 & 0,1840 & 0,0992 & 0,0370 & 0,1850 & 0,3890 \\
\hline Evidenciação Social & 2013 & 0,0702 & 0,0508 & 0,0000 & 0,0714 & 0,2020 \\
(EVS) & 2014 & 0,0660 & 0,0443 & 0,0000 & 0,0536 & 0,1790 \\
& 2015 & 0,0588 & 0,0424 & 0,0000 & 0,0476 & 0,1550 \\
& 2016 & 0,0570 & 0,0435 & 0,0000 & 0,0476 & 0,1670 \\
Evidenciação Total & 2017 & 0,0693 & 0,0611 & 0,0238 & 0,0476 & 0,2020 \\
(EVT) & 2013 & 0,0940 & 0,0669 & 0,0000 & 0,0906 & 0,2540 \\
& 2014 & 0,1080 & 0,0610 & 0,0000 & 0,1090 & 0,2030 \\
& 2015 & 0,0971 & 0,0575 & 0,0000 & 0,0906 & 0,2170 \\
& 2016 & 0,0945 & 0,0569 & 0,0000 & 0,0942 & 0,2100 \\
& 2017 & 0,1140 & 0,0603 & 0,0290 & 0,1090 & 0,2170 \\
\hline
\end{tabular}

Fonte: Dados da pesquisa.

Extrai-se que, durante o período 2013-2016, houve empresas que não divulgaram informações de práticas ambientais e sociais. Quanto à média da dimensão total, o ano de 2017 foi o que apresentou maior média de evidenciação. Isso pode ser explicado pela média da EVA, uma vez que o ano de 2017 apresentou a maior média de evidenciação.

A Tabela 9 apresenta os resultados dos modelos de regressão múltipla do tipo Pooled OLS, cuja variável dependente é o índice de evidenciação total (EVT). Ao todo foram gerados cinco modelos. No modelo (1), que contempla todas as variáveis, observa-se que a proporção de conselheiros independentes (INDEP) mostra-se significante a 5\% e positivo; e a atividade do conselho de administração (ATIV_CADM) apresenta-se significante a 1\% e negativo. Nos modelos (2) a (5), as variáveis independentes são testadas separadamente. No modelo (2), a variável INDEP se mantém significante a $5 \%$ e positiva; e a variável ATIV_CADM se mantém negativa e significante a $10 \%$ no modelo (5). As variáveis proporção de mulheres no conselho de administração (MLR_CADM) e idade média dos conselheiros (IM_CADM) não apresentaram significância nos modelos (3) e (4), respectivamente. Quanto às variáveis de controle, verifica-se que tamanho do conselho (TAM_CADM) apresenta-se significante em todos os modelos analisados. Observa-se nos modelos 
(1), (2), (3) e (5), significância estatística a 5\% na variável tamanho da empresa (TAM), e somente no modelo (4) o nível de significância foi de $10 \%$. Outra variável que demonstrou relevância estatística foi a alavancagem (ALAVAN) a 10\% de significância nos modelos (1) e (5).

Tabela 9 - Regressão múltipla do tipo pooled $O L S$ para a evidenciação total

\begin{tabular}{|c|c|c|c|c|c|c|c|c|c|c|}
\hline \multirow{2}{*}{ Variáveis independentes } & \multicolumn{10}{|c|}{ Evidenciação Total (EVT) } \\
\hline & (1) & & (2) & & (3) & & (4) & & (5) & \\
\hline INDEP & 0,0385 & $* *$ & 0,0349 & $* *$ & & & & & & \\
\hline MLR_CADM & 0,0090 & & & & $-0,0170$ & & & & & \\
\hline IM_CADM & $-0,0002$ & & & & & & $-0,0001$ & & & \\
\hline ATIV_CADM & $-0,0009$ & $* * *$ & & & & & & & $-0,0008$ & $* * *$ \\
\hline TAM_CADM & 0,0026 & $* * *$ & 0,0023 & $* * *$ & 0,0022 & $* * *$ & 0,0021 & $* *$ & 0,0025 & $* * *$ \\
\hline TAM & 0,0080 & $* *$ & 0,0068 & $* *$ & 0,0060 & $* *$ & 0,0061 & $*$ & 0,0069 & $* *$ \\
\hline ALAVAN & 0,0252 & $*$ & 0,0146 & & 0,0159 & & 0,0149 & & 0,0258 & $*$ \\
\hline SETOR & SIM & & SIM & & SIM & & SIM & & SIM & \\
\hline ANO & SIM & & SIM & & SIM & & SIM & & SIM & \\
\hline _CONS & $-0,0382$ & & $-0,0354$ & & $-0,0072$ & & $-0,0035$ & & $-0,0155$ & \\
\hline Observações & 190 & & 190 & & 190 & & 190 & & 190 & \\
\hline $\mathrm{F}$ & 9,45 & & 12,15 & & 11,43 & & 10,79 & & 10,69 & \\
\hline p-value & 0,0000 & & 0,0000 & & 0,0000 & & 0,0000 & & 0,0000 & \\
\hline $\mathrm{R}^{2}$ & 0,3636 & & 0,3457 & & 0,3313 & & 0,3308 & & 0,3456 & \\
\hline
\end{tabular}

Níveis de significância: $*<0,1 ; * *<0,05 ; * * *<0,01$.

Fonte: Dados da pesquisa.

Com relação à evidenciação ambiental (EVA), observa-se na Tabela 10, que os resultados nos modelos (6) e (7) indicam que a proporção de conselheiros independentes (INDEP) se apresenta significante a $10 \%$ e positivo. O modelo (6) mostra, ainda, que a atividade do conselho de administração é negativa e significante a $1 \%$.

De forma semelhante a EVT, observa-se que as variáveis proporção de mulheres no conselho de administração (MLR_CADM) e idade média dos conselheiros (IM_CADM) não apresentaram significância estatística nos modelos (6 e 8) e (6 e 9), respectivamente. As variáveis de controle tamanho do conselho de administração e tamanho da empresa demonstraram significância estatística nos modelos (6) a (9). A variável alavancagem apresentou significância a 5\% no modelo (6).

Tabela 10 - Regressão múltipla do tipo pooled $O L S$ para a evidenciação ambiental

\begin{tabular}{|c|c|c|c|c|c|c|c|c|c|}
\hline \multirow{2}{*}{ Variáveis independentes } & \multicolumn{9}{|c|}{ Evidenciação Ambiental (EVA) } \\
\hline & (6) & & (7) & & (8) & & (9) & & (10) \\
\hline INDEP & 0,0521 & $*$ & 0,0462 & $*$ & & & & & \\
\hline MLR_CADM & $-0,0068$ & & & & $-0,0513$ & & & & \\
\hline IM_CADM & $-0,0003$ & & & & & & $-0,0001$ & & \\
\hline ATIV_CADM & $-0,0017$ & $* * *$ & & & & & & & $-0,0016$ \\
\hline TAM_CADM & 0,0053 & $* * *$ & 0,0045 & $* * *$ & 0,0045 & $* * *$ & 0,0042 & $* *$ & 0,0050 \\
\hline TAM & 0,0138 & $* *$ & 0,0114 & $* *$ & 0,0105 & $* *$ & 0,0105 & $*$ & 0,0121 \\
\hline ALAVAN & 0,0552 & $* *$ & 0,0331 & & 0,0361 & & 0,0334 & & 0,0558 \\
\hline SETOR & SIM & & SIM & & SIM & & SIM & & SIM \\
\hline ANO & SIM & & SIM & & SIM & & SIM & & SIM \\
\hline _CONS & $-0,1165$ & & $-0,1083$ & & $-0,0720$ & & $-0,0657$ & & $-0,0883$ \\
\hline Observações & 190 & & 190 & & 190 & & 190 & & 190 \\
\hline $\mathrm{F}$ & 12,25 & & 15,32 & & 14,51 & & 13,54 & & 14,07 \\
\hline p-value & 0,0000 & & 0,0000 & & 0,0000 & & 0,0000 & & 0,0000 \\
\hline $\mathrm{R}^{2}$ & 0,4323 & & 0,4094 & & 0,4025 & & 0,4009 & & 0,4212 \\
\hline
\end{tabular}

Níveis de significância: $*<0,1 ; * *<0,05 ; * * *<0,01$.

Fonte: Dados da pesquisa.

Na Tabela 11, relativo à evidenciação social (EVS), a proporção de conselheiros independentes mostra-se significante a $5 \%$ e positiva nos modelos (11) e (12). Diferente do 
apresentado na EVT e EVA, a variável atividade do conselho de administração não demonstrou significância. De forma semelhante aos resultados de EVT e EVA, as variáveis MLR_CADM e IM_CADM não apresentaram significância.

Tabela 11 - Regressão múltipla do tipo pooled $O L S$ para a evidenciação social

\begin{tabular}{|c|c|c|c|c|c|c|}
\hline \multirow{2}{*}{ Variáveis independentes } & \multicolumn{6}{|c|}{ Evidenciação Social (EVS) } \\
\hline & (11) & & (12) & (13) & (14) & (15) \\
\hline INDEP & 0,0297 & $* *$ & 0,0275 & $* *$ & & \\
\hline MLR_CADM & 0,0192 & & & 0,0050 & & \\
\hline IM_CADM & $-0,0001$ & & & & $-0,0001$ & \\
\hline ATIV_CADM & $-0,0003$ & & & & & $-0,0002$ \\
\hline TAM_CADM & 0,0009 & & 0,0009 & 0,0007 & 0,0007 & 0,0008 \\
\hline TAM & 0,0042 & $*$ & 0,0037 & 0,0031 & 0,0032 & 0,0034 \\
\hline ALAVAN & 0,0059 & & 0,0026 & 0,0028 & 0,0029 & 0,0065 \\
\hline SETOR & SIM & & SIM & SIM & SIM & SIM \\
\hline ANO & SIM & & SIM & SIM & SIM & SIM \\
\hline _CONS & 0,0122 & & 0,0114 & 0,0345 & 0,0365 & 0,0313 \\
\hline Observações & 190 & & 190 & 190 & 190 & 190 \\
\hline $\mathrm{F}$ & 3,51 & & 3,93 & 3,36 & 3,39 & 3,37 \\
\hline p-value & 0,0000 & & 0,0000 & 0,0000 & 0,0000 & 0,0000 \\
\hline $\mathrm{R}^{2}$ & 0,1694 & & 0,1642 & 0,1482 & 0,1482 & 0,1508 \\
\hline
\end{tabular}

Níveis de significância: $*<0,1 ; * *<0,05 ; * * *<0,01$.

Fonte: Dados da pesquisa.

Quanto às variáveis de controle, diferente dos resultados apresentados em EVT e EVA, somente no modelo (11), o tamanho da empresa apresentou significância a $10 \%$ e positiva. Os valores dos coeficientes de determinação $\left(\mathrm{R}^{2}\right)$ nos modelos de (11) a (15) apresentam valores mais baixos, quando comparados a EVT e EVA. A Tabela 12 apresenta uma síntese dos resultados da pesquisa relacionados com as suas respectivas hipóteses:

Tabela 12 - Síntese dos resultados obtidos para a evidenciação total

\begin{tabular}{|c|c|c|}
\hline Relacionamento & Hipótese & Resultado \\
\hline INDEP $\rightarrow$ EVT & $\mathrm{H}_{1}$ : Efeito Positivo & Nuportada \\
\hline MLR_CADM $\rightarrow$ EVT & $\mathrm{H}_{2}:$ Efeito Positivo & Não suportada \\
\hline IM_CADM $\rightarrow$ EVT & $\mathrm{H}_{3}:$ Efeito Positivo & Suportada \\
\hline ATIV_CADM $\rightarrow$ EVT & $\mathrm{H}_{4}:$ Efeito Negativo & \\
\hline
\end{tabular}

Fonte: Elaborado a partir dos dados da pesquisa.

De modo geral, é aceita a influência positiva da proporção de conselheiros independentes na evidenciação de informações de caráter ambiental e social. Com relação a atividade do conselho de administração, verificou-se que influencia negativamente na evidenciação total e evidenciação ambiental. Dessa forma, são aceitas as Hipóteses 1 e 4, e rejeitam-se as Hipóteses 2 e 3, uma vez que, os resultados obtidos não apresentaram significância estatística para suportá-las.

\section{DISCUSSÃO}

\subsection{Papel da estrutura do conselho de administração na evidenciação de práticas ambientais e sociais}

A pesquisa confirma a influência da diversidade do conselho de administração na evidenciação de RSC. Os resultados indicam que, conselhos de administração que possuem uma maior proporção de conselheiros independentes estão mais propensos a um direcionamento voltado para a evidenciação de práticas ambientais e sociais. Ao longo dos anos de 2013 a 2017, o aumento na proporção de conselheiros independentes nas empresas listadas na B3 pode configurar uma 
redução nos conflitos de agência. Espera-se que um conselho de administração com maior proporção de conselheiros independentes seja mais eficiente ao monitorar as tarefas que envolvem a gestão dos riscos ambientais e sociais, além de apresentar níveis superiores de transparência. Dessa forma, incentivam um maior nível de divulgação voluntária por meio de um aumento nos investimentos em RSC (Jamali, Safieddine, \& Rabbath, 2008). Os conselheiros independentes apresentam uma conduta desvinculada de interesses, o que pode ocasionar no fornecimento de feedback mais objetivo sobre o desempenho organizacional. De acordo com Jizi (2017) e Jizi et al. (2014), esse tipo de conselheiro está mais inclinado a desenvolver a cidadania corporativa e definir agendas de RSC. Os resultados da pesquisa estão alinhados com os achados de Liao, Luo e Tang (2015), os quais afirmam que uma maior participação de conselheiros independentes garante a legitimidade organizacional.

A pesquisa também indica que a maior atividade do conselho de administração, representada pelo número de reuniões anuais, pode refletir negativamente na evidenciação de RSC. O conselho de administração pode necessitar de um maior número de reuniões quando a estratégia organizacional não consegue ser bem implementada, ou quando assuntos essenciais não são tratados como prioridade. Resultados semelhantes foram encontrados por Haji (2013), que afirma que uma elevada frequência de reuniões do conselho de administração pode ser um indicativo de um baixo desempenho social corporativo. $\mathrm{O}$ alto custo das reuniões do conselho de administração pode também interferir na elaboração de relatórios de RSC (Ahmad et al., 2017; Vafeas, 1999).

Os resultados da pesquisa indicaram, ainda, a não significância da influência da proporção de mulheres e da idade média dos membros do conselho de administração sobre a evidenciação de RSC. No tocante ao número de mulheres, por meio da estatística descritiva, observa-se a escassa representatividade de mulheres nos conselhos de administração em empresas brasileiras. Uma pesquisa realizada pelo IBGC (2016), constatou que o Brasil permanece em um patamar médio de $7 \%$ de mulheres nos conselhos de administração. Dessa forma, a voz feminina no conselho parece perder "espaço" ao tentar implantar e evidenciar práticas sociais e ambientais.

Com relação à idade média dos membros do conselho de administração, observou-se que gira em torno de 55 anos, com um baixo desvio padrão. Esse perfil etário dos conselheiros é semelhante ao estudo realizado pelo IBGC (2016). Nesse sentido, os membros do conselho de administração apresentam idades próximas, o que indica que podem concentrar valores semelhantes relacionados com a importância (ou não) das práticas ambientais e sociais, e que são refletidos na evidenciação de RSC.

\subsection{Desafios das empresas brasileiras para o avanço da evidenciação de práticas ambientais e sociais}

A pesquisa revela desafios para a ampliação da transparência e evidenciação de RSC. O crescimento na proporção de conselheiros independentes influenciará de maneira positiva a evidenciação de práticas ambientais e sociais nas empresas brasileiras. Os dados coletados para as 73 empresas listadas na B3 indicam que a proporção de conselheiros independentes no conselho de administração teve avanços significativos. Em 2013, a participação de conselheiros independentes era, em média, de 26\%. Em 2017 atingiu o patamar de 41\%. Este avanço também foi demonstrado em pesquisa realizada pelo IBGC (2016) e Spencer Stuart (2018). Rêgo, Vasconcelos e Santos (2018) indicaram que no ano de 2014, a proporção de conselheiros independentes no conselho de administração apresentava média de $21 \%$. Nesse sentido, verifica-se que as empresas brasileiras estão conseguindo ultrapassar as exigências mínimas impostas para o Novo Mercado ou Nível 2 de governança corporativa, que exige um percentual mínimo de $20 \%$ de conselheiros independentes (Spencer Stuart, 2018).

Outro desafio para as empresas brasileiras envolve a redução da atividade do conselho de administração. As empresas brasileiras apresentam uma média maior no total de reuniões anuais $(16,7)$, quando comparado com outros países, a exemplo de empresas da Malásia $(5,1)$ e da Alemanha $(6,1)$ (Ahmad et al., 2017; Dienes \& Velte, 2016). Com relação a alguns setores, também se observa 
esta diferença. Por exemplo, os bancos chineses apresentam média anual de 7,1 reuniões e as 350 maiores organizações listadas da Forbes de 1992 realizam, em média, 7,5 reuniões anuais (Liang, Xu, \& Jiraporn, 2013; Vafeas, 1999).

Empresas latino-americanas apresentam, comumente, uma alta concentração acionária e pertencem a grandes grupos familiares (Rossetti \& Andrade, 2012). No Brasil, é comum haver sobreposição das atividades de gestão com as atividades no conselho de administração. Isso resulta em conflitos e ineficiências na tomada de decisões estratégicas durante as reuniões do conselho de administração (Ahmad et al., 2017). Existe, portanto, um alto risco de o conselho de administração envolver-se indevidamente na gestão da empresa, que é de atribuição da diretoria executiva.

No que tange a diversidade de gênero, a pesquisa indica uma baixa representatividade feminina nos conselhos de administração de empresas brasileiras. Pesquisas apontam que a presença feminina nos conselhos de administração varia entre 7,9\% a 9,4\% (Dani, Picolo, \& Klann, 2019; IBGC, 2016), enquanto a média internacional se encontra na faixa de 24,1\% (Spencer Stuart, 2018). Apesar da preocupação com a diversidade de gênero no conselho de administração ter sido exposta no Projetos de Lei $n^{\circ} 112 / 2010$ e $n^{\circ} 7179 / 2017$, o Brasil ainda se encontra em $26^{\circ}$ lugar quanto à ocupação de mulheres em assentos efetivos do conselho de administração (Costa, Sampaio, \& Flores, 2019).

Nas empresas brasileiras, a idade média dos conselheiros é de aproximadamente 56 anos (Spencer Stuart, 2018) e caracteriza-se pela baixa dispersão etária entre os membros do conselho (Prudêncio, Forte, Crisóstomo, Vasconcelos, \& Rocha, 2019). O IBGC (2016) indica que o conselho de administração deve ser constituído considerando-se a diversidade de conhecimentos, experiências, comportamentos, aspectos culturais, faixa etária e gênero. Distintos valores e visões de mundo podem influenciar a tomada de decisão, de modo a beneficiar a atuação responsável das empresas.

\section{CONCLUSÃO}

A pesquisa permite ampliar o conhecimento sobre o relacionamento da governança corporativa, especificamente de práticas ligadas à composição do conselho de administração, com a responsabilidade social corporativa. Nesse sentido, o estudo mostra que a estrutura do conselho de administração, no que diz respeito à proporção de conselheiros independentes e o número de reuniões, influenciam a evidenciação de práticas ambientais e sociais. A presença de conselheiros independentes reduz os conflitos de agência e incentiva a transparência. Quanto à atividade do conselho, o maior número de reuniões pode indicar que decisões que poderiam ser tomadas pela gestão da empresa, possivelmente, estejam sendo levadas para o conselho de administração.

Há expectativas de que as corporações tenham responsabilidade sobre os interesses dos stakeholders. Apoiando-se na Teoria da Agência, a pesquisa confirma a importância de práticas de governança corporativa no alinhamento dos interesses da gestão com as demandas dos stakeholders, e que garantam uma adequada comunicação e transparência no tratamento das questões ambientais e sociais. O estudo também contribui ao ampliar as discussões sobre a governança corporativa e a responsabilidade social corporativa no contexto dos países em desenvolvimento. Diferente de nações desenvolvidas, a responsabilidade social corporativa praticada por empresas no Brasil caracteriza-se como menos formalizada e mais filantrópica, permitindo que as empresas experimentem novas formulações e definições de sustentabilidade.

A pesquisa apresenta limitações que devem ser reconhecidas. O tamanho da amostra pode representar uma limitação, uma vez que foram selecionadas apenas as empresas listadas na B3 (Brasil, Bolsa, Balcão), que divulgaram relatórios de RSC no formato GRI-G4, no período 2013-2017. No entanto, deve-se destacar que a amostra contempla empresas que divulgaram informações no mesmo padrão e que possibilita comparações entre si. Outra possível limitação envolve o curto período de tempo analisado, em virtude da indisponibilidade de indicadores de evidenciação ambiental e social, em uma longa série temporal. 
Embora se reconheçam limitações empíricas, este trabalho contribui para a pesquisa sobre o relacionamento da composição do conselho de administração com a evidenciação de práticas ambientais e sociais. Estudos comparativos com empresas listadas em outras bolsas de valores, usando o modelo econométrico proposto, podem ampliar o entendimento da importância de uma adequada governança corporativa.

\section{AGRADECIMENTOS}

Ao Editor Prof. Roberto Carlos Klann e aos Revisores Anônimos por suas valiosas contribuições para a melhoria da versão inicial do artigo. Ao CNPq pelo financiamento ao Projeto de Pesquisa, e a FUNCAP pela concessão das bolsas de estudos.

\section{REFERÊNCIAS}

Ahmad, N. B. J., Rashid, A., \& Gow, J. (2017). Board meeting frequency and corporate social responsibility (CSR) reporting: Evidence from Malaysia. Corporate Board: role, duties and composition, 13(1), 8799.

Amran, A., Lee, S. P., \& Devi, S. S. (2014). The influence of governance structure and strategic corporate social responsibility toward sustainability reporting quality. Business Strategy and the Environment, 23(4), 217-235.

Basuony, M. A., Mohamed, E. K., \& Samaha, K. (2018). Board structure and corporate disclosure via social media: an empirical study in the UK. Online Information Review. 42(5), 595-614.

Bear, S., Rahman, N., \& Post, C. (2010). The impact of board diversity and gender composition on corporate social responsibility and firm reputation. Journal of Business Ethics, 97(2), 207-221.

Campbell, K., \& Mínguez-Vera, A. (2008). Gender diversity in the boardroom and firm financial performance. Journal of Business Ethics, 83(3), 435-451.

Costa, L., Sampaio, J. D. O., \& Flores, E. S. (2019). Diversidade de gênero nos conselhos administrativos e sua relação com desempenho e risco financeiro nas empresas familiares. Revista de Administração Contemporânea, 23(6), 721-738.

Dani, A. C., Picolo, J. D., \& Klann, R. C. (2019). Gender influence, social responsibility and governance in performance. RAUSP Management Journal, 54(2), 154-177.

De La Cuesta, M., \& Valor, C. (2013). Evaluation of the environmental, social and governance information disclosed by Spanish listed companies. Social Responsibility Journal. 9(2), 220-240.

Dienes, D., \& Velte, P. (2016). The impact of supervisory board composition on CSR reporting. Evidence from the German two-tier system. Sustainability, 63(8), 1-20.

Fernández-Gago, R., Cabeza-García, L., \& Nieto, M. (2016). Corporate social responsibility, board of directors, and firm performance: an analysis of their relationships. Review of Managerial Science, 10(1), 85-104.

Ferrero-Ferrero, I., Fernández-Izquierdo, M. Á., \& Muñoz-Torres, M. J. (2015). Integrating sustainability into corporate governance: an empirical study on board diversity. Corporate Social Responsibility and Environmental Management, 22(4), 193-207.

Fischer, T. M., \& Sawczyn, A. A. (2013). The relationship between corporate social performance and corporate financial performance and the role of innovation: Evidence from German listed firms. Journal of Management Control, 24(1), 27-52.

Font, X., Walmsley, A., Cogotti, S., McCombes, L., \& Häusler, N. (2012). Corporate social responsibility: The disclosure-performance gap. Tourism Management, 33(6), 1544-1553.

García-Sánchez, I. M., Suárez-Fernández, O., \& Martínez-Ferrero, J. (2019). Female directors and impression management in sustainability reporting. International Business Review, 28(2), 359-374.

Giannarakis, G. (2014). Corporate governance and financial characteristic effects on the extent of corporate social responsibility disclosure. Social Responsibility Journal. 10(4), 569-590.

Global Reporting Initiative (2013). G4 - Diretrizes para relato de sustentabilidade. Manual de implementação. Amsterdam: GRI.

Global Reporting Initiative (2015). G4 - Diretrizes para relato de sustentabilidade. Princípios para relato e conteúdo padrão. Amsterdam: GR 
Guidry, R. P., \& Patten, D. M. (2010). Market reactions to the first-time issuance of corporate sustainability reports. Sustainability Accounting, Management and Policy Journal. 1(1), 33-50.

Hafsi, T., \& Turgut, G. (2013). Boardroom diversity and its effect on social performance: Conceptualization and empirical evidence. Journal of Business Ethics, 112(3), 463-479.

Haji, A. A. (2013). Corporate social responsibility disclosures over time: evidence from Malaysia. Managerial Auditing Journal. 28(7), 647-676.

Harjoto, M. A., \& Jo, H. (2011). Corporate governance and CSR nexus. Journal of Business Ethics, 100(1), 45-67.

Harrison, J. S., Bosse, D. A., \& Phillips, R. A. (2010). Managing for stakeholders, stakeholder utility functions, and competitive advantage. Strategic Management Journal, 31(1), 58-74.

Helfaya, A., \& Moussa, T. (2017). Do board's corporate social responsibility strategy and orientation influence environmental sustainability disclosure? UK evidence. Business Strategy and the Environment, 26(8), 1061-1077.

Hillman, A. J., \& Dalziel, T. (2003). Boards of directors and firm performance: Integrating agency and resource dependence perspectives. Academy of Management Review, 28(3), 383-396.

Hu, M., \& Loh, L. (2018). Board governance and sustainability disclosure: A cross-sectional study of Singapore-listed companies. Sustainability, 10(7), 2578.

Instituto Brasileiro de Governança Corporativa - IBGC (2015). Código das melhores práticas de governança corporativa. São Paulo.

Instituto Brasileiro de Governança Corporativa - IBGC (2016). Perfil dos conselhos de administração. São Paulo.

Jamali, D., Safieddine, A. M., \& Rabbath, M. (2008). Corporate governance and corporate social responsibility synergies and interrelationships. Corporate Governance: An International Review, 16(5), 443-459.

Jensen, M. C. (1993). The modern industrial revolution, exit, and the failure of internal control systems. The Journal of Finance, 48(3), 831-880.

Jizi, M. (2017). The influence of board composition on sustainable development disclosure. Business Strategy and the Environment, 26(5), 640-655.

Jizi, M. I., Salama, A., Dixon, R., \& Stratling, R. (2014). Corporate governance and corporate social responsibility disclosure: Evidence from the US banking sector. Journal of Business Ethics, 125(4), 601-615.

Liang, Q., Xu, P., \& Jiraporn, P. (2013). Board characteristics and Chinese bank performance. Journal of Banking \& Finance, 37(8), 2953-2968.

Liao, L., Luo, L., \& Tang, Q. (2015). Gender diversity, board independence, environmental committee and greenhouse gas disclosure. The British Accounting Review, 47(4), 409-424.

Modiba, E. M., \& Ngwakwe, C. C. (2017). Women on the corporate board of directors and corporate sustainability disclosure. Corporate Board: Role, Duties, 32-37.

Muttakin, M. B., Khan, A., \& Mihret, D. G. (2018). The effect of board capital and CEO power on corporate social responsibility disclosures. Journal of Business Ethics, 150(1), 41-56.

Naciti, V. (2019). Corporate governance and board of directors: The effect of a board composition on firm sustainability performance. Journal of Cleaner Production, 237(1), 1-7.

Post, C., Rahman, N., \& Rubow, E. (2011). Green governance: Boards of directors' composition and environmental corporate social responsibility. Business \& Society, 50(1), 189-223.

Prudêncio, P. A., Forte, H. C., Crisóstomo, V. L., Vasconcelos, A. C., \& Rocha, T. L. C. G. (2019). Efeitos da diversidade do conselho de administração e da diretoria executiva na responsabilidade social corporativa. Anais do International Conference In Accounting, São Paulo, SC, Brasil, 19.

Pucheta-Martínez, M. C., Bel-Oms, I., \& Olcina-Sempere, G. (2018a). Commitment of independent and institutional women directors to corporate social responsibility reporting. Business Ethics: A European Review, 28(3), 290-304.

Pucheta-Martínez, M. C., Bel-Oms, I., \& Olcina-Sempere, G. (2018b). The association between board gender diversity and financial reporting quality, corporate performance and corporate social responsibility disclosure: A literature review. Academia Revista Latinoamericana de Administración. 31(1), 177-194.

Rao, K., \& Tilt, C. (2016). Board composition and corporate social responsibility: The role of diversity, gender, strategy and decision making. Journal of Business Ethics, 138(2), 327-347. 
Rêgo, B. P., de Vasconcelos, A. C., \& dos Santos, J. G. C. (2018). Efeitos da Estrutura de Governança Corporativa e das Características Institucionais no Disclosure Socioambiental. Revista Eletrônica de Ciência Administrativa, 17(3), 344-372.

Reverte, C. (2016). Corporate social responsibility disclosure and market valuation: evidence from Spanish listed firms. Review of Managerial Science, 10(2), 411-435.

Roberts, R. W. (1992). Determinants of corporate social responsibility disclosure: An application of stakeholder theory. Accounting, Organizations and Society, 17(6), 595-612.

Rossetti, J. P., \& Andrade, A. (2012) Governança corporativa: fundamentos, desenvolvimento e tendências. (6a ed). São Paulo: Atlas.

Said, R., Zainuddin, Y. H., \& Haron, H. (2009). The relationship between corporate social responsibility disclosure and corporate governance characteristics in Malaysian public listed companies. Social Responsibility Journal. 5(2), 212-226.

Soares, R. A., Pinheiro, A. B., de Abreu, M. C. S., \& Marino, P. D. B. L. P. (2018). Efeito do sistema financeiro na evidenciação socioambiental de empresas em países emergentes e desenvolvidos. Enfoque: Reflexão Contábil, 37(2), 21-35.

Soares, R. A., de Abreu, M. C. S., Marino, P. D. B. L. P., \& Rebouças, S. M. D. P. (2018). Avaliação hierárquica da influência do país, setor e empresa na evidenciação da responsabilidade social corporativa. Contabilidade Vista \& Revista, 29(2), 96-121.

Stuart, S. (2018). 2018 Turkey Spencer Stuart Board Index. São Paulo: Spencer Stuart.

Torchia, M., Calabrò, A., Huse, M., \& Brogi, M. (2010). Critical mass theory and women directors' contribution to board strategic tasks. Corporate Board: Role, Duties \& Composition, 6(3).

Vafeas, N. (1999). Board meeting frequency and firm performance. Journal of Financial Economics, 53(1), 113-142.

Van Marrewijk, M. (2003). Concepts and definitions of CSR and corporate sustainability: Between agency and communion. Journal of Business Ethics, 44(2), 95-105.

Zajac, E. J., \& Westphal, J. D. (1996). Who shall succeed? How CEO/board preferences and power affect the choice of new CEOs. Academy of Management Journal, 39(1), 64-90. 